\title{
Correction to: Association of bowel radiation dose-volume with skeletal muscle loss during pelvic intensity-modulated radiotherapy in cervical cancer
}

\author{
Jie Lee ${ }^{1,2} \cdot$ Jhen-Bin Lin ${ }^{3} \cdot$ Meng-Hao Wu ${ }^{1} \cdot$ Chih-Long Chang ${ }^{2,4} \cdot$ Ya-Ting Jan ${ }^{5} \cdot$ Fang-Ju Sun $^{6} \cdot$ Yu-Jen Chen ${ }^{1}$ \\ Published online: 25 November 2021 \\ ๑) Springer-Verlag GmbH Germany, part of Springer Nature 2021
}

Correction to: Supportive Care in Cancer (2021) 29:5497-5505

https://doi.org/10.1007/s00520-021-06131-x

The correct Table 4 should be the below:

Publisher's Note Springer Nature remains neutral with regard to jurisdictional claims in published maps and institutional affiliations.

The original article can be found online at https://doi.org/10.1007/ s00520-021-06131-x.

Jie Lee

sinus.5706@mmh.org.tw

1 Department of Radiation Oncology, MacKay Memorial Hospital, 92, Section 2, Chung Shan North Road, Taipei 10449, Taiwan

2 Department of Medicine, MacKay Medical College, New Taipei City, Taiwan

3 Department of Radiation Oncology, Changhua Christian Hospital, Changhua, Taiwan

4 Department of Obstetrics and Gynecology, MacKay Memorial Hospital, Taipei, Taiwan

5 Department of Radiology, MacKay Memorial Hospital, Taipei, Taiwan

6 Department of Medical Research, MacKay Memorial Hospital, Taipei, Taiwan 
Table 4 Cox proportional hazards model for overall survival

\begin{tabular}{|c|c|c|c|c|}
\hline \multirow[b]{2}{*}{ Variable } & \multicolumn{2}{|l|}{ Univariable } & \multicolumn{2}{|l|}{ Multivariable } \\
\hline & Hazard ratio $(95 \% \mathrm{CI})$ & $p$-value & Hazard ratio $(95 \% \mathrm{CI})$ & $p$-value \\
\hline \multicolumn{5}{|l|}{ Age } \\
\hline$<70$ years & Reference & & Reference & \\
\hline$\geq 70$ years & $1.63(0.96-2.76)$ & 0.07 & $1.23(0.68-2.25)$ & 0.50 \\
\hline \multicolumn{5}{|l|}{ ECOG performance status } \\
\hline 0 & Reference & & - & - \\
\hline 1 & $1.21(0.52-2.83)$ & 0.66 & - & - \\
\hline \multicolumn{5}{|l|}{ FIGO stage } \\
\hline IB-II & Reference & & Reference & \\
\hline III-IVA & $1.80(1.04-3.10)$ & 0.04 & $1.97(1.09-3.57)$ & 0.03 \\
\hline \multicolumn{5}{|l|}{ PLNs involvement } \\
\hline Negative & Reference & & - & - \\
\hline Positive & $1.21(0.72-2.05)$ & 0.47 & - & - \\
\hline \multicolumn{5}{|l|}{ Pathology } \\
\hline Squamous cell carcinoma & Reference & & Reference & \\
\hline Adenocarcinoma & $3.19(1.71-5.94)$ & $<0.001$ & $2.16(1.03-4.50)$ & 0.04 \\
\hline SCC-Ag level, continuous & $1.00(0.99-1.01)$ & 0.50 & - & - \\
\hline \multicolumn{5}{|l|}{ NLR } \\
\hline$\leq 3$ & Reference & & Reference & \\
\hline$>3$ & $2.42(1.42-4.13)$ & 0.001 & $2.45(1.32-4.54)$ & 0.005 \\
\hline Pre-treatment SMI & $0.97(0.933-1.003)$ & 0.08 & $0.99(0.95-1.04)$ & 0.70 \\
\hline \multicolumn{5}{|l|}{ SMI change } \\
\hline SMI maintain & Reference & & Reference & \\
\hline SMI loss $(\geq-10.0 \%)$ & $8.02(4.70-13.69)$ & $<0.001$ & $5.73(2.90-11.34)$ & $<0.001$ \\
\hline Pre-treatment SMD & $1.008(0.98-1.04)$ & 0.60 & - & - \\
\hline \multicolumn{5}{|l|}{ SMD change } \\
\hline SMD maintain & Reference & & Reference & \\
\hline SMD loss $(\geq-10.0 \%)$ & $3.66(2.17-6.15)$ & $<0.001$ & $1.83(0.94-3.56)$ & 0.08 \\
\hline Pre-treatment TATI & $0.994(0.988-1.001)$ & 0.07 & $0.998(0.989-1.006)$ & 0.58 \\
\hline \multicolumn{5}{|l|}{ TATI change } \\
\hline TATI maintain & Reference & & Reference & \\
\hline TATI loss $(\geq-10.0 \%)$ & $1.99(1.18-3.37)$ & 0.01 & $1.20(0.64-2.22)$ & 0.57 \\
\hline
\end{tabular}

Abbreviations: CI, confidence interval; ECOG, Eastern Cooperative Oncology Group; FIGO, International Federation of Gynecology and Obstetrics; NLR, neutrophil-lymphocyte ratio; PLN, pelvic lymph node; SCC-Ag, squamous cell carcinoma antigen; SMD, skeletal muscle density; SMI, skeletal muscle index; TATI, total adipose tissue index 